\title{
Mutual Coupling Reduction Between Axial-Mode Helical Antennas using Single-Negative (SNG) Magnetic Metamaterials
}

\author{
M. M. Bait-Suwailam*a and O.M. Ramahi \\ Department of Electrical and Computer Engineering, University of Waterloo, 200 University Ave. W., Waterloo, Ontario \\ N21, 3G1, Canada \\ Received 12 May 2009; accepted 19 April 2010

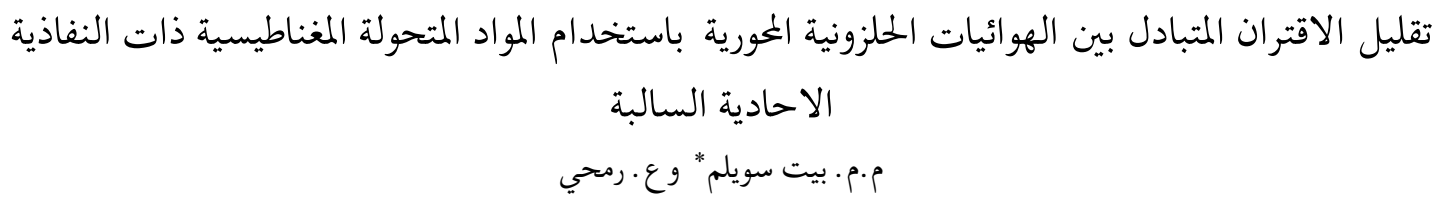

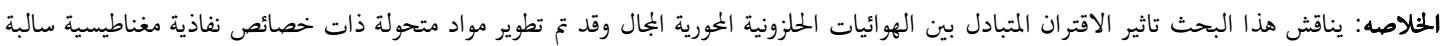

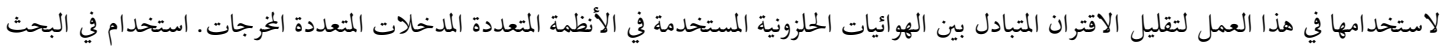

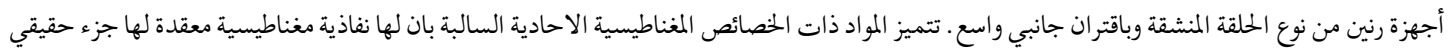

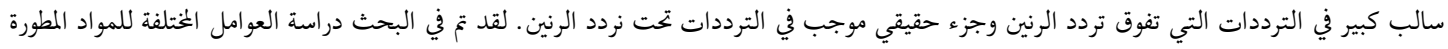

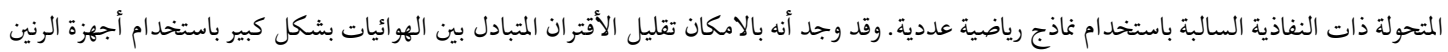

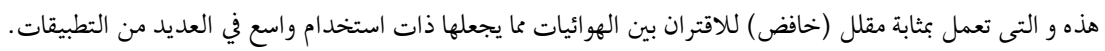 \\ المفردات المفتاحية: المواد الممغنطة اصطناعيا، الجمال العوري، الهوائيات الحلزونية، المواد المتحولة، الأقتران المتبادل .
}

\begin{abstract}
This paper investigates the mutual coupling effects between axial-mode helical antenna arrays. Single-negative (SNG) magnetic metamaterials are developed and used in this work in order to reduce mutual coupling between axial-mode helical antennas used in multiple-input multiple-output systems. The inclusions considered here are composed of broadside coupled split-ring resonators. The magnetic permeability of those SNG inclusions have complex effective magnetic permeability response over a frequency band with high negative real part above the resonance and positive real part below the resonance. The SNG resonators are inserted between closely-spaced axial-mode helical antenna elements. Effective responses of the constitutive parameters of the developed magnetic inclusions are incorporated within the numerical models. It is shown that mutual coupling between the antenna elements can be reduced significantly by incorporating such magnetic inclusions. The SNG magnetic resonators work as antenna decoupler, and thus can be applied in a variety of antenna applications.
\end{abstract}

Keywords: Artificial magnetic materials, Axial-mode, Helical antennas, Metamaterial, and mutual coupling

\section{Introduction}

A widely used high profile antenna type is the helical antenna. Axial-mode helical antenna has become popular due to its low-cost, light weight, wide band, and is used in many applications requiring circularly polarized $(\mathrm{CP})$ radiation patterns and high gain. Axialmode helices typically have high gain depending on

$\overline{\text { * Corresponding author's e-mail:mbaitsuw@engmail.uwaterloo.ca }}$ the number of turns and other factors (Kruas 1988). Axial-mode helical antennas have their maximum radiation along the axial direction, unlike the normalmode helices where their maximum radiation goes broadside to the helix axis.

For high gain applications, multiple antenna elements are employed in an array. Enhancement in the performance and capacity of the antenna system can 
be achieved in comparison to a single-input single-output system (SISO) (Foschini and Gans, 1998). Such mechanism, or enhancement, is possible if the antenna elements are well-decorrelated since information can be sent through multiple elements at the transmitter and/or the receiver side more efficiently. Therefore the performance of antenna arrays or multiple-input multiple-output (MIMO) systems can be degraded when the antenna elements are in close proximity. The degradation is due to near-field effects, diffraction from finite-ground planes and strong inductive and capacitive coupling between the elements (Balanis, 2005 and Vaughan and Andersen, 1987).

Mutual coupling in antenna arrays becomes significant when placing the radiators close to each other. Nakano et al. 1984 has investigated the possibility of reducing the mutual coupling between two short (1.5 turns) identical helical antennas by rotating one helix with respect to another. Mutual impedance between bifilars of a quadrifilar helix antenna has been previously studied in (Amin and Chahill, 2005). Signal correlation between two normal-mode helical antennas has been studied in (Hui et al. 2004). The correlation coefficient was expressed based on the mutual impedances of the antenna system.

The performance of MIMO antenna elements can be evaluated using the envelope correlation parameter which can be derived from scattering parameters. In many outdoor and indoor wireless communication environments, there are many obstacles and scatterers, like buildings, mobile terminals, and offices...etc. A problem that is encountered in such environments is multipath fading. This fading phenomenon limits the performance and capacity of the antenna system. One of the ways to combat that multipath fading is to use multiple antennas at either transmit, receive or both ends. In MIMO systems, more than two antennas are often employed. Thus, the correlation between any two antennas within the array is often required and need to be kept as low as possible. In (Blanch et al. 2003), the envelope correlation is derived from the scattering parameters of an antenna system. The correlation between different planar inverted F-antenna (PIFA) elements has been studied in (Thaysen and Jakobsen, 2006). It is observed that by using the scattering parameters to compute the envelope correlations in the antenna array is more cost effective in comparison to using the far-field radiation pattern method.

To the best of our knowledge, no analysis has been conducted for axial-mode helical antenna arrays incorporating metamaterials as antenna decouplers. In this paper, electromagnetic coupling between closelyspaced axial-mode helical antennas with single-negative (SNG) magnetic metamaterials is numerically analyzed and discussed. Mutual coupling and enve- lope correlation between two helical antenna elements are considered. Other important antenna parameters such as return loss, axial ratio, and field patterns are also presented.

SNG metamaterials can have negative permittivity or negative permeability values over a certain frequency band. One way of realizing SNG metamaterials is to use split-ring resonators (SRR) (Pendry et al. 1999 and Marques et al. 1996). Once these inclusions are excited using proper polarization, an induced electric current develops on the inclusions. If a large number of inclusions are arranged either periodically or aperiodically, the inclusions behave as magnetic dipole arrays. Those dipole elements result in having negative effective permeability $\mu_{\text {eff }}$, over a certain frequency band. This in turn prevents the existence of real propagating modes within the SNG metamaterials. Thus, it is plausible that an SNG material can block electromagnetic energy radiated by one antenna element from being transmitted to a nearby antenna element within an array system. This implies that the ensemble of resonators, comprising the metamaterial, act as a magnetic shielding layer.

This paper is organized as follows. Section II gives a conspectus of the theory and motivation behind using single-negative (SNG) magnetic metamaterials as magnetic shielding layer. The design methodology for the magnetic materials considered in this work is also discussed, with emphasis on the numerical characterization of the magnetic metamaterials. Numerical results are presented and discussed in Section III. Brief summary and concluded remarks are given in Section IV.

\section{Single-negative (SNG) Magnetic Meta- materials as Magnetic Decoupling Layer}

Figure 1 shows a classification of materials based on their constitutive parameters, the electrical permittivity $\varepsilon$, and magnetic permeability $\mu$. In microwave frequencies, $\varepsilon$ and $\mu$ are both positive for natural materials, which can be termed double-positive (DPS) materials. Material with negative permittivity and negative permeability (DNG), however, cannot exist naturally over microwave frequencies and engineered artificial materials are used to realize such materials (Pendry et al. 1999; Marques et al. 1996 and Pendry et al. 1996).

Although there are fundamental differences in the propagation and refraction properties of DPS and DNG (Veselago, 1968), both types of materials can transmit electromagnetic waves. From an electromagnetic interference (EMI) point of view, inserting a slab of DPS or DNG material between two high profile antennas does not have a significant effect. An inter- 


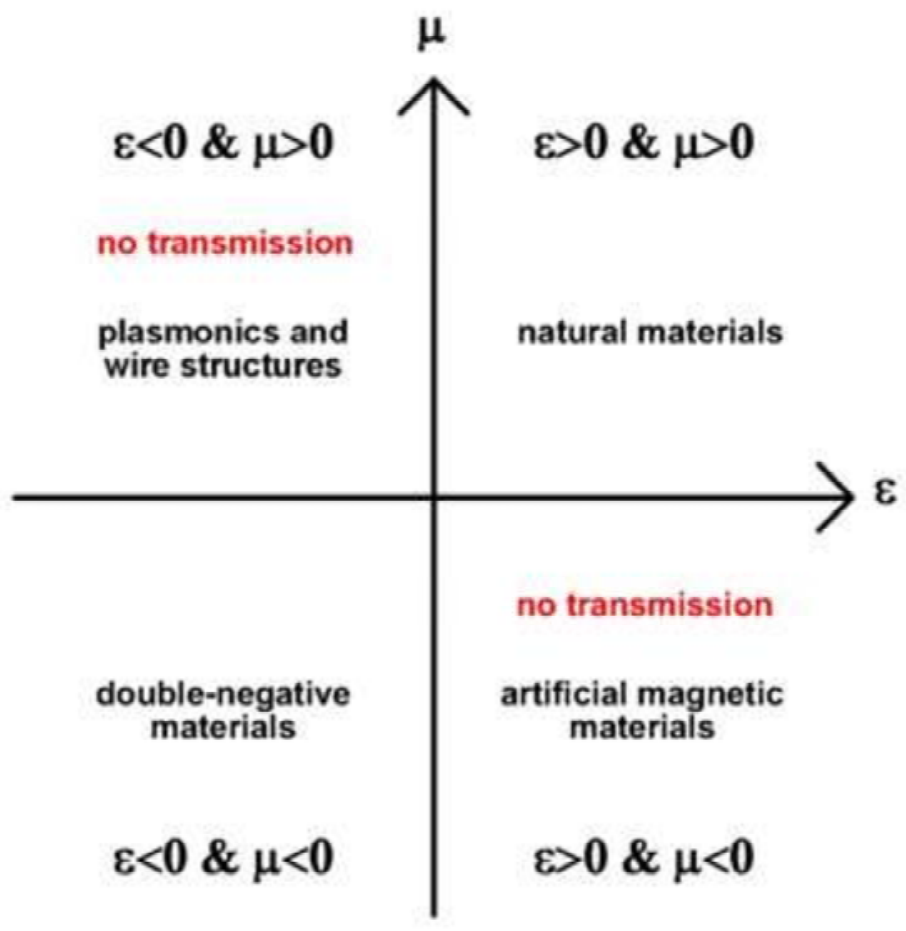

Figure 1. Materials classification based on their constitutive paramaters $\varepsilon-\mu$

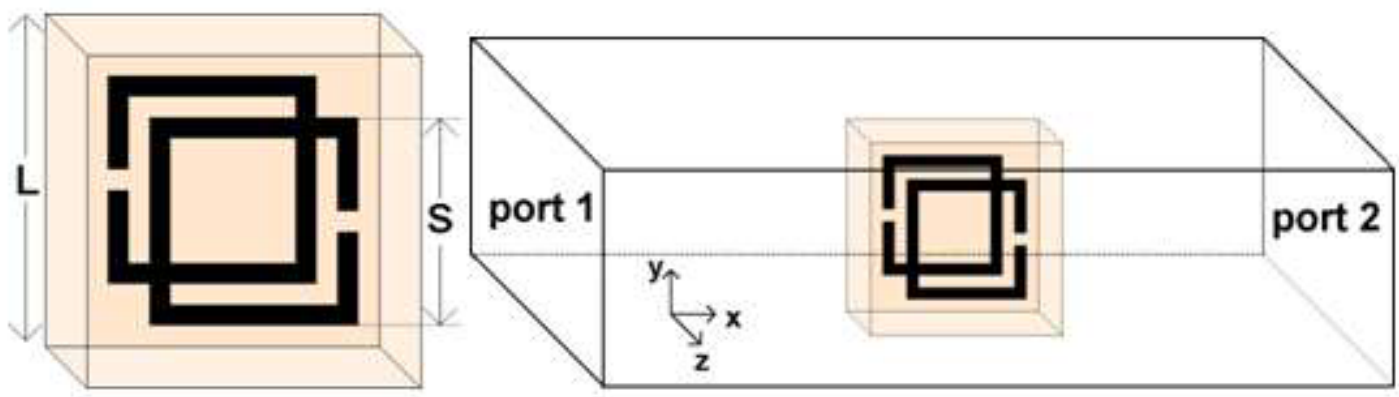

(a)

(b)

Figure 2. The developed single-negative magnetic metamaterials based on the (BC-SRRs): (a) Splitring resonator (SRR) unit cell with its dimensions, (b) waveguide structure used for the characterization of MNG metamaterials. Note the E-Field points in $y$-direction, H-field is in $z$-direction, and the propagation is $x$-direction

esting phenomenon of strong relevance to the wide range of problems where electromagnetic interference is of concern is the elimination of propagation when one of the constitutive parameters, either $\varepsilon_{\text {eff }}$ or $\mu_{\text {eff }}$, is negative. This can be seen from the second and fourth quadrants of the classification of materials, the $\varepsilon-\mu$ domain shown in Fig. 1. In such a scenario, transmission of electromagnetic energy would cease since purely evanescent waves exist within the SNG medium. Therefore, a single-negative (SNG) metamaterials would be expected to reduce the mutual coupling and decouple the closely-spaced antenna elements.

\subsection{Single-negative (SNG) Magnetic Metamate- rials Design}

The single-negative (SNG) metamaterial considered in this work has a real positive effective electrical permittivity, $\varepsilon_{\text {eff, }}$ and a complex effective magnetic permeability, $\mu_{\text {eff }}$ with a negative real part. The magnetic permeability is negative over the frequency band, where we desire decreased coupling between the antennas. In (Smith et al. 2000; Maslo0vski et al. 2005 and Erentok et al. 2005), several types of SNG metamaterial designs were discussed. In this work, we consider one of the popular types, namely the rectangular split-ring resonators (SRRs). Figure 2a shows a unit cell of the single-negative magnetic inclusion. It is 
made of two concentric copper rings, each interrupted by a small gap. This capacitive gap in addition to the capacitance between the concentric rings strongly influences the resonance of the SRR inclusion. As such, it is possible to realize a resonant magnetic inclusions with dimensions of only $\lambda / 10$ of the operating wavelength (ie. in this work, dimensions of roughly $\lambda / 15$ are considered as compared to a closed ring which requires a dimension of $\lambda / 4$.)

If an alternating magnetic field is incident upon the SRR plane, the SRR behaves as a parallel inductorcapacitor $(L C)$ circuit. This $L C$ circuit exhibits a resonant response at a frequency $\omega_{\mathrm{m}}=1 / \sqrt{ } L C$, associated with a resonant current circulating around the rings. These resonant circular currents give rise to a resonant magnetic dipole moments, thus SRRs inclusions can be characterized by resonant effective permeability, which is usually followed by a negative permeability regime (ie. on the high-frequency side of SRRs resonance). This lumped LC behavior is valid since the dimensions of the SRR inclusions are much smaller than the operating wavelength. One way to trace the negative permeability regime in SRRs or even in metamaterials in general is through transmission measurements or simulations, where this regime appears as a transmission dip. The characterization and retrieval of SNG magnetic metamaterials are considered next.

The model adopted in our work is based on the retrieval extraction method reported in (Chen 2004). Basically, one have to compute the scattering parameters of a unit cell SRR inclusion, from which the effective refractive index $\boldsymbol{n}$ and impedance $\boldsymbol{Z}$ are first computed. The effective permittivity and permeability $\mu$ are then calculated from $\mu=n \boldsymbol{Z}$ and $\varepsilon=n / \boldsymbol{Z}$.

In order to numerically characterize the SRR inclusions, an air-filled waveguide with a unit cell positioned at the center of the waveguide is used as shown in Fig. 2b. Notice that the cell is positioned such that the incident magnetic field is perpendicular to the inclusion's trace/surface. The model mimics a transverse electromagnetic mode in both ports of the waveguide (see Fig. 2b), with top and bottom sides of the air-filled waveguide assigned as perfect electric conducting (PEC) walls (ie. walls orthogonal to the $y$ axis), while its sides as perfect magnetic (PMC) walls (ie. walls orthogonal to the $z$-axis).

Initial design dimensions of the SRR inclusion were estimated numerically using the model discussed above. Next, the optimized dimensions were obtained such that the inclusion resonance takes place at the middle of the frequency band of interest. The SRR rings considered here have equal sides of lengths $S=$ $12 \mathrm{~mm}$. The SRR rings with opposite cut openings are etched on the sides of a dielectric substrate (Rogers RO4350, $\varepsilon_{r}=3.48, \tan \delta=0.004$ ) having a thickness of $0.762 \mathrm{~mm}$. The cut (gaps) within the metallic rings is $1 \mathrm{~mm}$. The size of the dielectric substrate, $L$, (see Fig. 2b) is $16 \mathrm{~mm}$, which is much less than the operating wavelength at a frequency of $1.24 \mathrm{GHz}$. The SRR rings are made of copper with a thickness of $20 \mu \mathrm{m}$. In this work, 8 SRR inclusion pairs are etched on both sides of the dielectric substrate. The periodicity of the magnetic inclusions is an important parameter in the design of such inclusions, and it needs to be much smaller than the operating wavelength in order to realize a homogenous medium for the SRR resonators. The periodicity, or separation distance between each strip of the developed magnetic inclusions (see Fig. 2), corresponds to an electrical thickness of $\lambda / 15$ at SRR's resonance.

Figure 3 shows the scattering parameters, $S_{11}$ and $S_{21}$, computed using two commercially available fullwave electromagnetic solvers (Ansoft.com HFSS (ansoft.com), and CST Microwave Studio (MWS) (CST Microwave Studio, www.cst.com). Good agreement between the two solvers is achieved. The real and imaginary parts of the effective electric permittivity and magnetic permeability of the SRR resonators are then extracted using the retrieval method discussed above. The extracted parameters are shown in Fig. 4.

\subsection{Effective Parameters Approach}

Due to computational memory constraints, the effective response of the magnetic inclusions (see Fig. 4 ) is used in order to mimic the response of the $\mu$-negative magnetic inclusions. The artificial magnetic structures are in general inherently dispersive and anisotropic. The MNG materials considered in this work are based on the broadside-coupled BC-SRRs (Marques et al. 2002). The SRRs inclusions provide enhanced permeability only in the direction normal to the inclusions plane ( $z$-direction in Fig. 2b), and enhanced permittivity in the directions tangent to the plane ( $x$ - and $y$-directions). In this work, the orientation of the magnetic inclusions is assumed in xy-plane, as shown in Fig. 2b. In such a case, the effective permeability and permittivity of the magnetic structures can take the following tensor forms, respectively.

$$
\begin{aligned}
& \mu(\omega)=\mu_{O}\left[\begin{array}{lll}
1 & 0 & 0 \\
0 & 1 & 0 \\
0 & 0 & \mu_{z z}(\omega)
\end{array}\right] \\
& \varepsilon(\omega)=\varepsilon_{O}\left[\begin{array}{ccc}
\varepsilon_{x x}(\omega) & 0 & 0 \\
0 & \varepsilon_{y y}(\omega) & 0 \\
0 & 0 & \varepsilon_{r}
\end{array}\right],
\end{aligned}
$$

where $\varepsilon_{r}$ represents the permittivity of the host medium $\left(\varepsilon_{r}=3.48, \tan \delta=0.004\right)$.

It is instructive to note that within the numerical models, the effective permeability in the $(x, y, z)$ direc- 


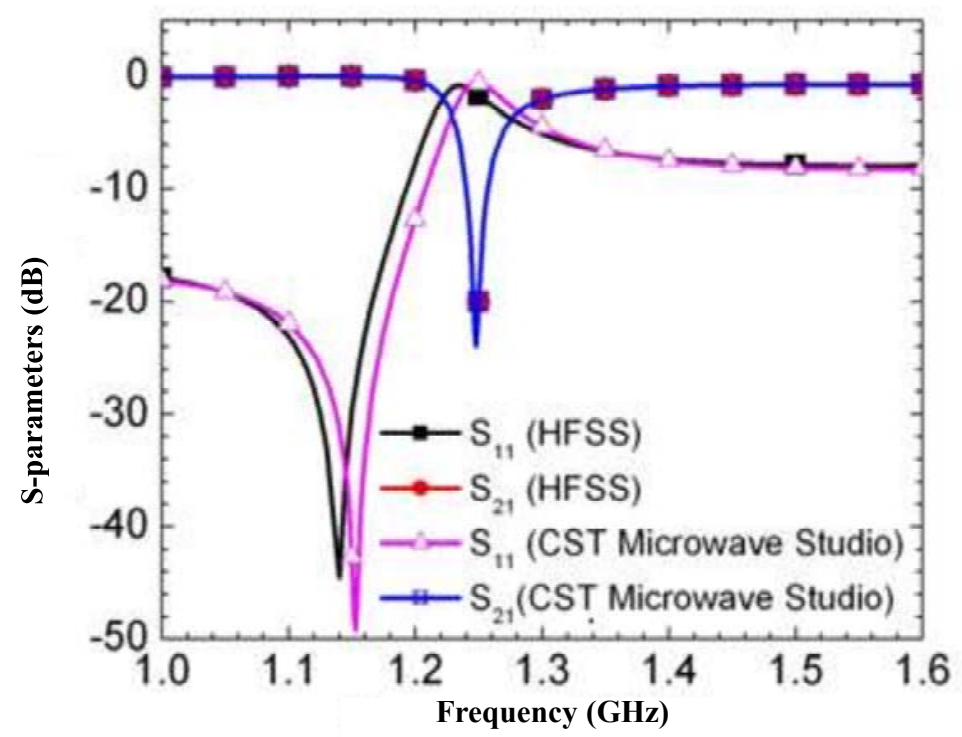

Figure 3. Scattering parameters of an SNG metamaterials' unit-cell extracted from the waveguide structure shown in Figure 2b

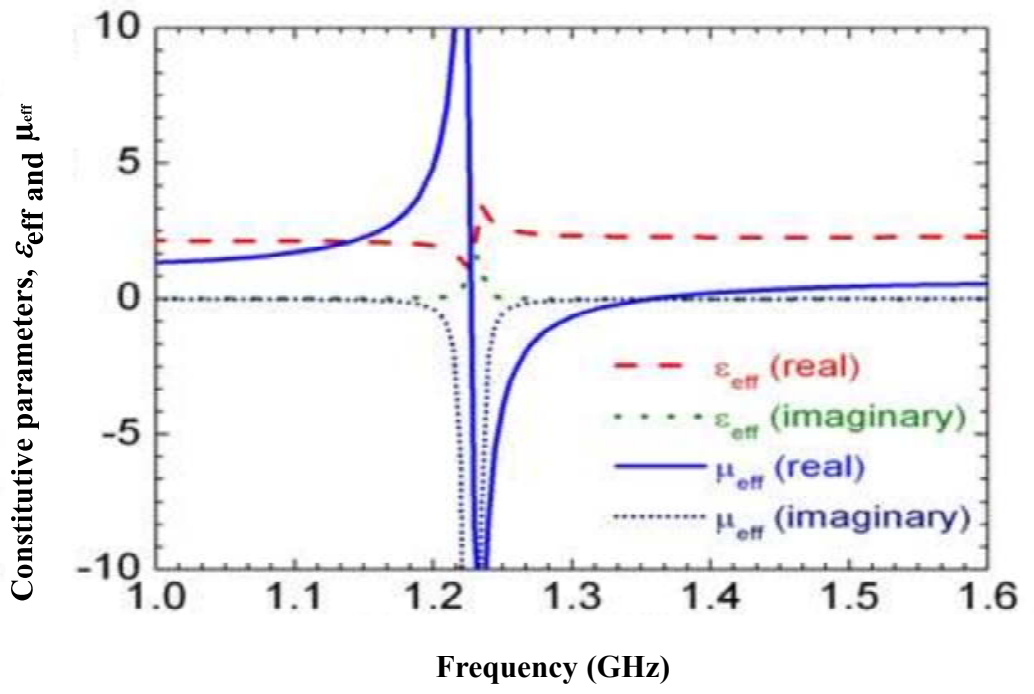

Figure 4. Effective response for the constitutive parameters of the SNG unit cell extracted using the retrieval method

tions correspond to $\left(1,1, \mu_{\mathrm{zz}}(\omega)\right)$, while the effective permittivity response corresponds to $\left(\varepsilon_{\mathrm{xx}}(\omega), \varepsilon_{\mathrm{yy}}(\omega)\right.$, $\left.\varepsilon_{\mathrm{r}}\right)$ in $(x, y, z)$ respectively, as can be seen from (1) and (2). The magnetic SNG inclusions are oriented in such a way that the external magnetic field would couple into them, (the external magnetic field is perpendicular to the magnetic inclusions).

\section{Numerical Results}

Figure 5 shows a simple sketch for a typical helical antenna. Basically, the antenna is in the form of a helix wire of a certain diameter; however, a strip wire can also be used for ease of integration and miniaturization. The antenna is excited from one end and radiate from the other open end. In this analysis, a strip of width, $w=3 \mathrm{~mm}$ is used as the radiating element. The antenna considered here is left-hand circularly polarized (LHCP). A helical antenna is usually specified by its diameter $(D)$, number of turns $(N)$, spacing between each two consecutive turns $(S)$, a pitch angle $(\alpha)$, and a wire turn length $(L)$. In this work, a helix with 4 turns is considered. The diameter of the helical antenna $D=0.32 \lambda_{0}$, spacing, $S=\lambda_{0} / 5$, and a circumference of $1 \lambda_{0}$ is used.

Figure 6 shows the HFSS model for a single axial- 


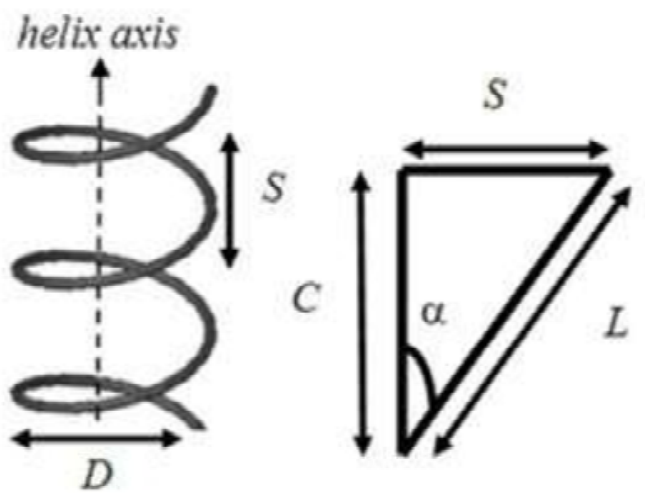

Figure 5. Design parameters of the helical antenna

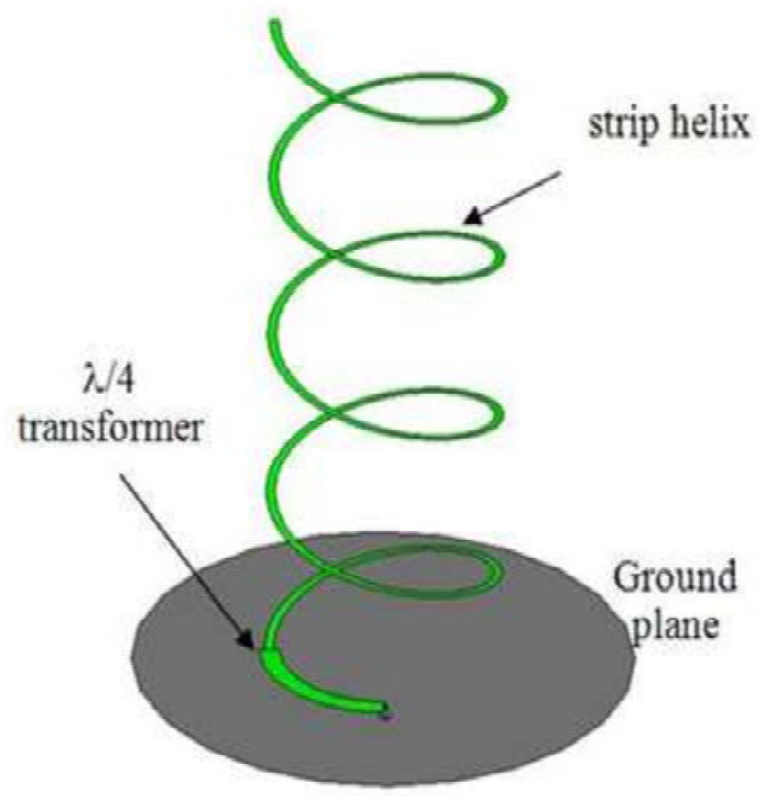

Figure 6. HFSS model for a single axial-mode helix antenna

mode helical antenna. A quarter-wavelength transformer was used to match a $50 \Omega$ SMA connector to the helix antenna. Figure 7 depicts the frequency response of the axial-ratio (AR) for the single helix antenna, where the axial-ratio remains within $3 \mathrm{~dB}$ over the antenna operating band. Thus, it quantifies the circular polarized waves generated out of the antenna.

Figure 8 shows the model setup used for the coupling analysis between two helical antenna elements separated by a distance of $0.5 \lambda^{0}$, where single-negative metamaterial strips (SNG inclusions) are inserted between the two antennas. A reference geometry comprising two helical antennas spaced by the same distance but without the SNG magnetic inclusions is used for comparison. A perfectly conducting (PEC) screen is investigated as well in order to quantify the performance of the proposed magnetic shielding screen. Note

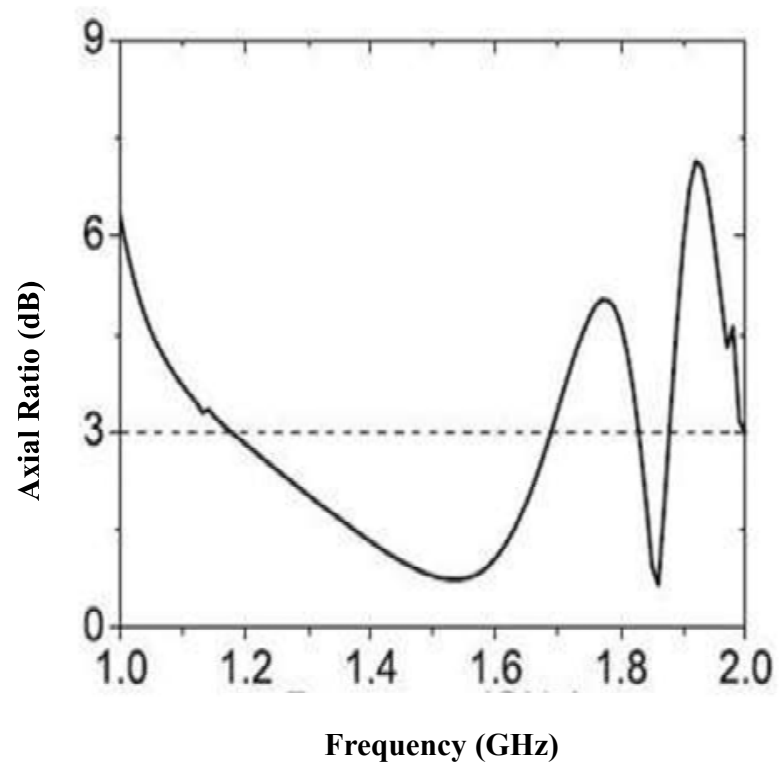

Figure 7. Frequency characteristics of axial-ratio in $\mathrm{dB}$ for the single helix antenna

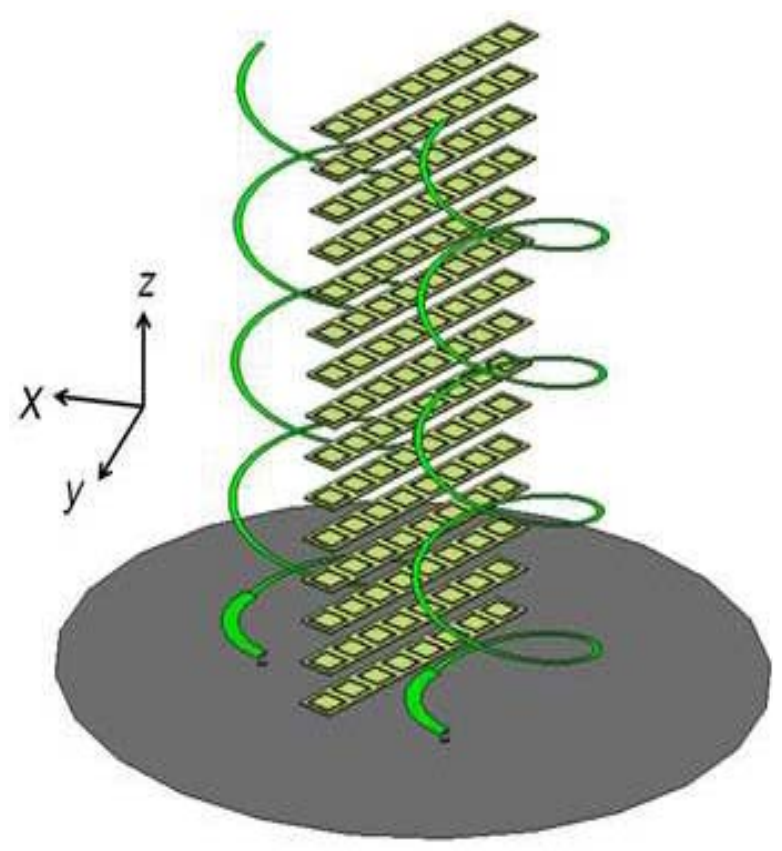

Figure 8. HFSS model for the two helical antennas with strips of single-negative metamaterials. Note that feed center-to-centre distance is $\lambda_{\mathrm{o}} / 2$

that the width of the PEC screen corresponds to the electrical thickness of the developed magnetic strips, $\lambda_{\mathrm{o}} / 16$.

Figure 9 shows the computed reflection coefficient, $S_{11}$, for the two helical antennas in free-space and when using single-negative magnetic inclusions. Good impedance matching is observed over a wide frequen- 


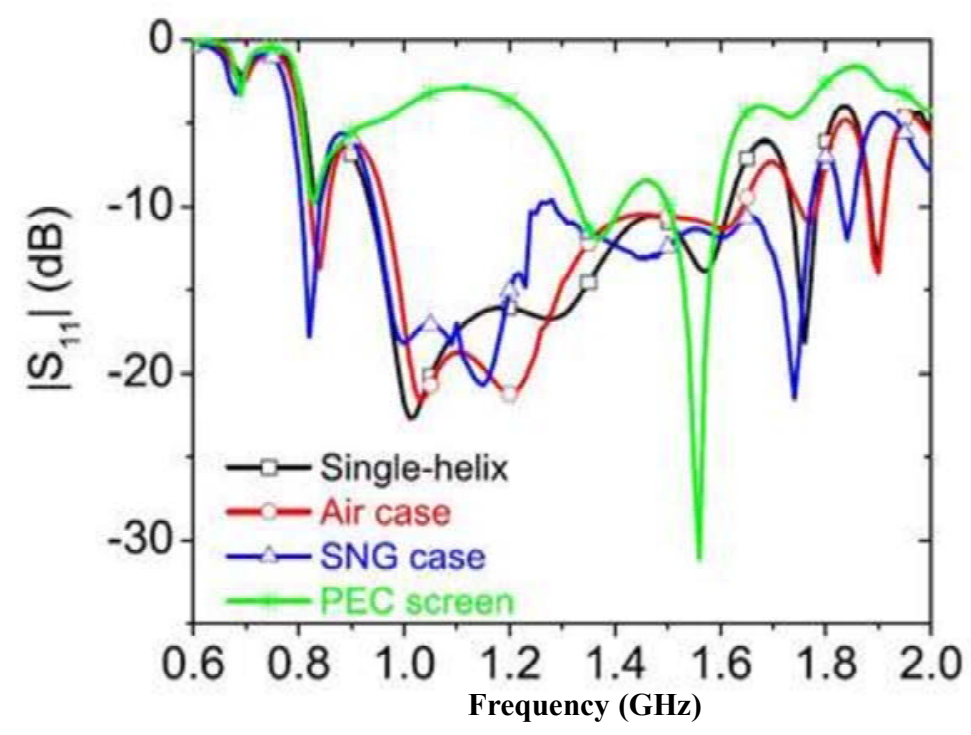

Figure 9. Magnitude of $S_{I I}$ for the helical antenna system with and without SNG metamaterials

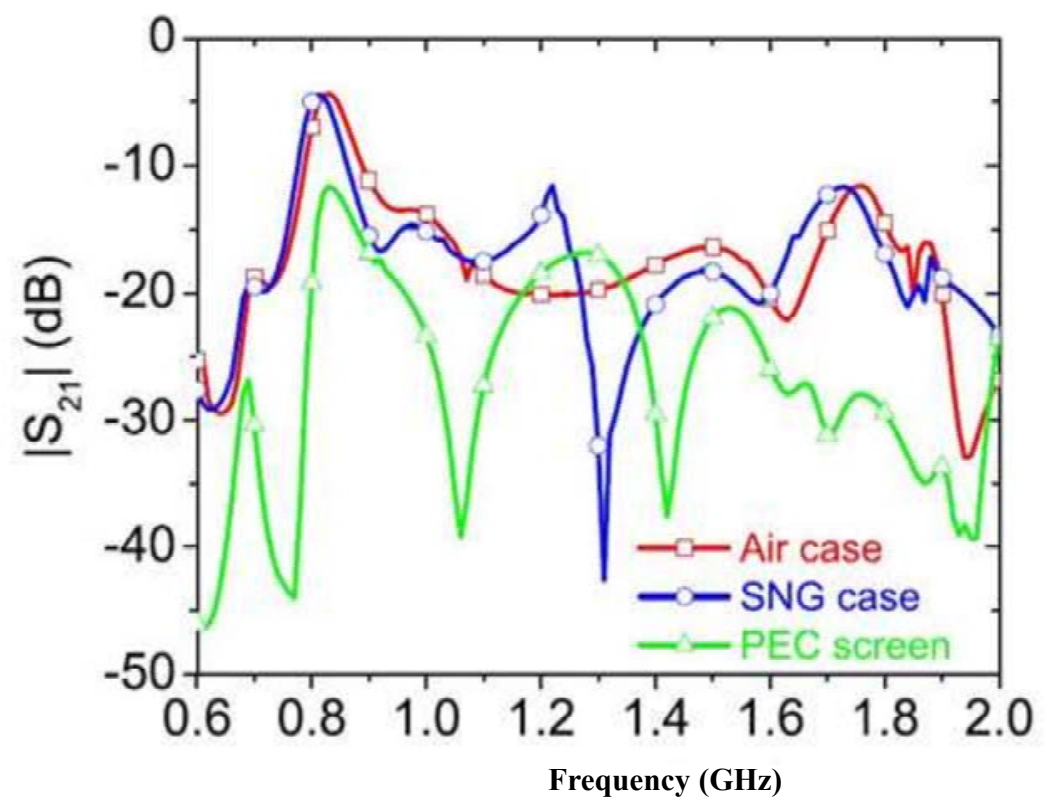

Figure 10. Magnitude of $S_{21}$ for the helical antenna system with and without SNG metamaterials

cy band of $\approx 54 \%$ for both cases with and without the magnetic inclusions. The impedance matching deteriorates for the PEC screen case over the antennas operating frequency, where total reflection from the PEC screen is expected.

The mutual coupling, $S_{21}$, between the two helical antennas with and without the SNG magnetic inclusions (see Fig. 8) is computed and shown in Fig. 10. A reduction of more than $20 \mathrm{~dB}$ is achieved while creating a slight shift in the resonance frequency (approximately $70 \mathrm{MHz}$ ). Thus, the coupling between the heli- cal antenna elements has been reduced over a frequency band from $1.26 \mathrm{GHz}$ up to $1.4 \mathrm{GHz}$. A noticeable dip of $S_{21}$ is observed at a frequency of $1.31 \mathrm{GHz}$. Moreover, helical antennas show less mutual coupling effects over the frequency band used in this analysis as compared to the monopole antennas studied in (BaitSuwailam et al. 2009). Nevertheless, the effect of the developed magnetic inclusions in enhancing the gain of the helical antennas is of concern in this work. More isolation between the helical antenna elements is attainable when using the PEC screen, as expected, 
although the use of the SNG magnetic inclusions shows higher reduction of mutual coupling around the resonance frequency of $1.31 \mathrm{GHz}$.

Next, the performance of antenna arrays applicable to MIMO systems is studied. In a rich scattering multipath environment, the correlation between the antenna elements can be evaluated using antennas far-field components (Vaughan and Andersen, 1987), mutual impedances between the antenna elements Derneryd and Kristensson, 2004 and Hui and Lui, 2008), or directly from the scattering parameters measured at the ports (Blanch et al. 2003 and Thaysen and Jakobsen, 2006). Under the assumptions of uniform incident waves along the azimuth plane, uncorrelated incident waves impinging from the elevation plane and good impedance matching, the envelope correlation between antenna elements can be calculated from the scattering parameters much more readily than from the far-field patterns. In this work, the use of scattering parameters is incorporated in the envelope correlation computation. The antenna system performance can be determined by the correlation matrix. For better performance, antennas should have low correlation, as well as high isolation between antenna elements.

For multiple lossless antennas consisting of N elements, the correlation matrix is related to the scattering parameters as

$$
\mathrm{C}=\mathrm{I}-\mathrm{S}^{\mathrm{H}} \mathrm{S} \text {, }
$$

and can be represented in a matrix form as

$$
C=\left[\begin{array}{l}
1-\left(\left|S_{11}\right|^{2}+\left|S_{21}\right|^{2}\right)-\left(S_{11}^{*} S_{12}+S_{21}^{*} S_{22}\right) \\
-\left(S_{12}^{*} S_{11}+S_{22}^{*} S_{21}\right) 1-\left(\left|S_{12}\right|^{2}+\left|S_{22}\right|^{2}\right)
\end{array}\right]
$$

where the superscript $H$ denotes the Hermitian of the scattering matrix $S$, and $I$ is the identity matrix. The auto- and cross-correlation parameters can be represented as $C_{i i}$ and $C_{i j}$, respectively, where $i$ and $j$ denotes the antenna elements ports. While the autocorrelation $C_{i i}$ quantifies the amount of radiated power from antenna $i$ (similarly for $C_{j j}$ ), the cross-correlation $C_{i j}$ gives insights into the cross-coupling between antenna elements $i$ and $j$.

The envelope correlation $\rho_{\mathrm{e}}$, is given as (Blanch et al. 2003).

$$
\rho_{e}(i, j, N)=\frac{\left|\sum_{n=1}^{N} S_{i, n}^{*} S_{n, j}\right|^{2}}{\prod_{k=i, j}\left[1-\sum_{n=1}^{N} S_{i, k}^{*} S_{n, k}\right]}
$$

In the case of a $2 \times 2$ MIMO antenna system $(N=$ $2)$, the envelope correlation between antenna element $i$ $=1$ and $j=2$ is given as

$$
\rho_{e}(1,2,2)=\frac{\left|S_{11}^{*} S_{12}+S_{21}^{*} S_{22}\right|^{2}}{\left[1-\left(\left|S_{11}\right|^{2}+\left|S_{21}\right|^{2}\right)\right]\left[1-\left(\left|S_{22}\right|^{2}+\left|S_{12}\right|^{2}\right)\right]}
$$

The envelope correlation between the helical antenna elements has been reduced (see Fig. 11) by almost $10 \mathrm{~dB}$ when using the magnetic inclusions. Figure 12 shows the autocorrelation, $C_{11}$, computed using the antennas scattering parameters. The antenna elements with SNG magnetic inclusions gave almost similar correlation to that of the air case. This is attributed to that the antenna elements with and without the SNG magnetic resonators have in average low mutual coupling. More reduction in the correlation is observed around $1.31 \mathrm{GHz}$, where reduction in mutual coupling is observed when using the SNG magnetic layer. The cross-correlation, $C_{21}$, results are shown in Fig. 13. The helical antennas with the SNG magnetic inclusions are well-decorrelated over a frequency band covering the antenna system resonance.

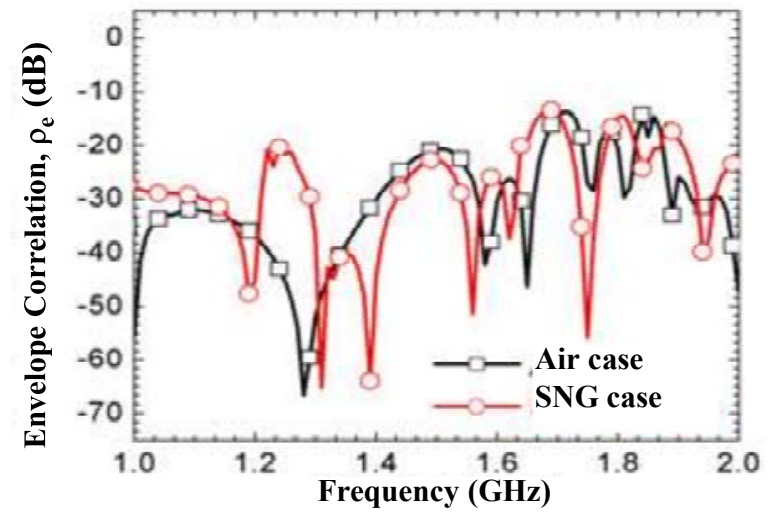

Figure 11. Envelope correlation, $\rho_{\mathrm{e}}$, for the helical antenna system with and without SNG magnetic metamaterials

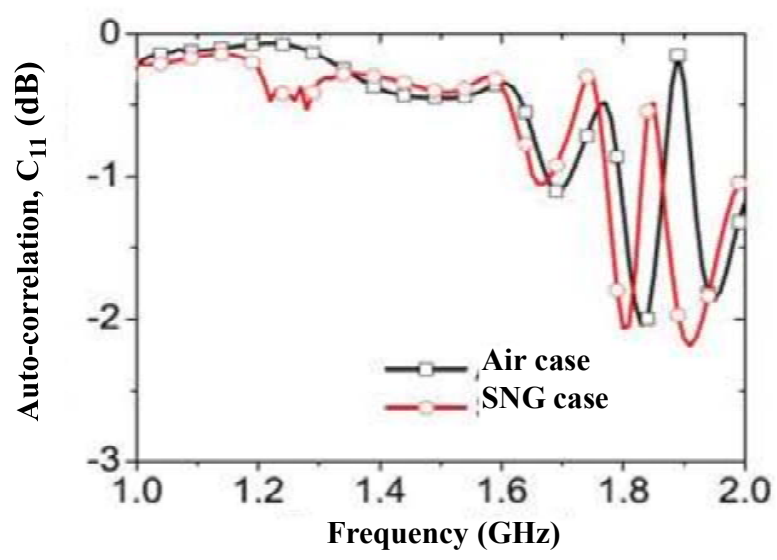

Figure 12. Computed auto-correlation for the helical antenna system with and without SNG magnetic metamaterials 


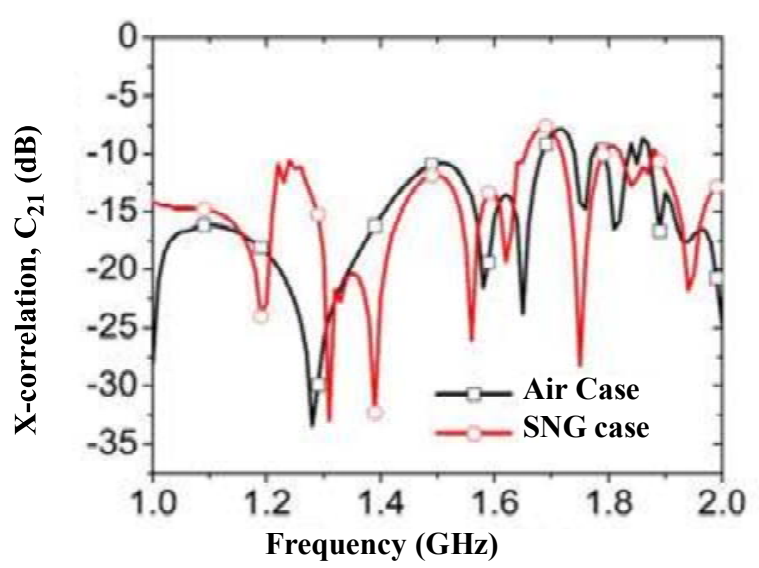

Figure 13. Computed cross-correlation for the helical antenna system with and without SNG magnetic metamaterials

Figure 14 shows the gain pattern in axial direction for the single helix antenna, the two helical antennas with and without the SNG magnetic inclusions. A gain of $9.8 \mathrm{~dB}$ is obtained for the single axial-mode helix antenna in the axial-direction (ie. $z$-direction in this analysis), while a gain of $12.8 \mathrm{~dB}$ is obtained for the two helices in free-space. The computed gain for the SRR case is $13.0 \mathrm{~dB}$. Thus, by placing the magnetic inclusions between the helical antenna elements, the gain has been increased, and the mutual coupling between the antenna elements has been reduced by almost $20 \mathrm{~dB}$ (see Fig. 10).

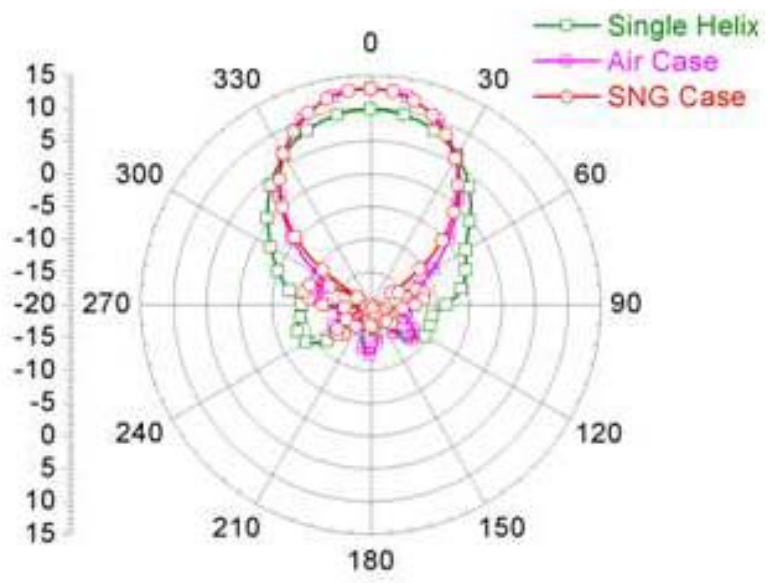

Figure 14. Far-field gain pattern in axial-direction for the single-helix, the two helices with and without the magnetic inclusions, computed at a frequency of 13.1 GHz

\section{Conclusions}

In this paper, mutual coupling between closelyspaced axial-mode helical antennas is investigated with particular focus on multiple-input multiple-out- put (MIMO) systems. Guidelines for the design and analysis of single-negative (SNG) magnetic resonators as antenna decoupler were discussed in details. The metamaterials constructed in this work have complex effective magnetic permeability, $\mu_{\text {eff }}$, with high negative real part above the enhanced resonance.

The developed magnetic inclusions show their effectiveness in terms of reducing the mutual coupling between the helical antenna elements and enhancing the gain of the helix antenna array. Numerical results show that an almost $20 \mathrm{~dB}$ reduction in mutual coupling between antenna elements is attainable. Moreover, good impedance matching is maintained when using the magnetic inclusions. The gain of the two axial-mode helical antenna system has increased as a result of inserting the magnetic resonators between the two radiators. Such a decoupling layer is an efficient magnetic shielding wall that can be advantageous in antenna applications and EMI problems.

\section{Acknowledgment}

This work was supported by Sultan Qaboos University of Oman, Research in Motion Inc., and the National Science and Engineering Research Council of Canada under the NSERC/RIM Industrial Research Chair and Discovery Programs.

\section{References}

Amin, M. and Cahill, R., 2005. "Mutual Impedance between Bifilars of a Quadrifilar Helix Antenna", in High Frequency Postgraduate Student Colloquium, pp. 66-69.

Ansoft HFSS, http://www.ansoft.com.

Bait-Suwailam, M.M., Boybay, M.S. and Ramahi, O.M., 2009, "Electromagnetic Coupling Reduction in High-Profile Antennas Using SingleNegative Metamaterials for MIMO Applications", in International Conference on Communication, Computer and Power (ICCCP), pp. 115-120.

Balanis, C.A., 2005, " Antenna Theory Analysis and Design", John Wiley and Sons Inc.

Blanch, S., Romeu, J. and Corbella, I., 2003, "Exact Representation of Antenna System Diversity Performance from Input Parameter Description", Electron. Lett., Vol. 39(9), pp. 705-707.

Chen, X., Grzegorczyk, T.M., Wu, B.I., Pacheco, J. and Kong, J.A., 2004, "Robust Method to Retrieve the Constitutive Effective Parameters of Metamaterials", Phys. Rev. E, Vol. 70(1), pp. 016 608.1-016 608.7.

CST Microwave Studio, http://www.cst.com.

Derneryd, A. and Kristensson, G., 2004, "Antenna Signal Correlation and its Relation to the 
Impedance Matrix”, Electron. Lett., Vol. 40(7), pp. 401-402.

Erentok, A., Luljak, P. and Ziolkowski, R., 2005, "Characterization of a Volumetric Metamaterial Realization of an Artificial Magnetic Conductor for Antenna Applications", IEEE Trans. Antennas Propag., Vol. 53(1), pp. 160-172.

Foschini, G.J. and Gans, MJ., 1998, "On Limits of Wireless Communications in a Fading Environment when Using Multiple Antennas", Wireless Personal Communications, Vol. 6(3), pp. 311-335.

Hui, H. and Lui, H.S., 2008, "Expression of Correlation Coefficient for Two Omindirectional Antennas using Conventional Mutual Impedances", Electron. Lett., Vol. 44(2), pp. 1177-1178.

Hui, H.T., Yong, W. and Toh, K., 2004, "Signal Correlation between Two Normal-mode Helical Antennas for Diversity Reception in a Multipath Environment", IEEE Trans. Antennas Propag., Vol. 52(2), pp. 572-577.

Kraus, J.D., 1988, " Antennas", 2nd ed. McGraw-Hill. Marques, R., Medina, F. and Rafii-El-Idrissi, R., 2002, "Role of Bianisotropy in Negative Permeability and Left-handed Metamaterials", Phys. Rev. B, Vol. 65, pp. 144 440-6.

Maslovski, S., Ikonen, P., Kolmakov, I., Tretyakov, S. and Kaunisto, M., 2005, "Artificial Magnetic Materials Based on the New Magnetic Particle: Metasolenoid", Progress in Electromagnetic Research, Vol. 54, pp. 61-81.
Nakano, H., Asaka, N. and Yamauchi, J., 1984, "Radiation Characteristics of Short Helical Antenna and its Mutual Coupling”, Electron. Lett., Vol. 20(5), pp. 202-204.

Pendry, J., Holden, A., Robbins, D. and Stewart, W., 1999, "Magnetism from Conductors and Enhanced Nonlinear Phenomena", IEEE Trans. Microw. Theory Tech., Vol. 47(11). pp. 20752084.

Pendry, J.B., Holden, A.J., Stewart, W.J. and Youngs, I., 1996. "Extremely Low Frequency Plasmons in Metallic Mesostructures", Phys. Rev. Lett., Vol. 76(25), pp. 4773-4776.

Smith, D.R., Padilla, W.J., Vier, D.C., NematNasser, S.C. and Schultz, S., 2000, "A Composite Medium with Simultaneously Negative Permeability and Permittivity", Phys. Rev. Lett., Vol. 84, pp. 4184-4187.

Thaysen, J. and Jakobsen, K., 2006, "Envelope Correlation in (N, N) MIMO Antenna Array from Scattering Parameters," Microwave and Opt. Tech. Letts., Vol. 48(5), pp. 832-834.

Vaughan, R. and Andersen, J., 1987, "Antenna Diversity in Mobile Communications," IEEE Transactions on Vehicular Technology, Vol. 36(4), pp. 149-172, Nov. 1987.

Veselago, V.G., 1968, "The Electrodynamics of Substances with Simulataneously Negative Values of $\varepsilon$ and $\mu$,", Soviet Physics Uspekhi, Vol. 10(4), pp. 509-514. 